\title{
CÓDIGOS TIPO: DERECHO A LA INFORMACIÓN Y PROTECCIÓN DE DATOS PERSONALES
}

\author{
María-del-Carmen Rodríguez-López
}

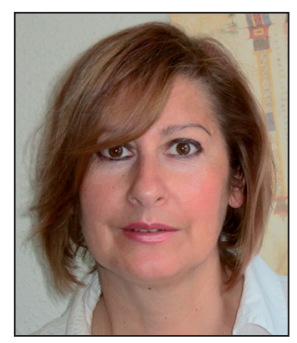

María-del-Carmen-Rodríguez-López, doctora por la Univ. de León, es profesora titular del Área de Biblioteconomía y Documentación, Depto. de Patrimonio Artístico y Documental, de la misma universidad, como responsable de las asignaturas de archivística, archivos administrativos, organización de fondos archivísticos y gestión de archivos. Imparte también docencia en el master en asesoría jurídica de la empresa, de la Facultad de Derecho, en la asignatura gestión documental del archivo de empresa. Entre sus líneas de investigación se encuentran: archivos y actas municipales, valor económico de la gestión del servicio de archivo de historias clínicas, sistemas de gestión del documento de archivo y protección de datos. Dirige el grupo de investigación Anaquel: Sistemas de información, documentación y archivo.

Área de Biblioteconomía y Documentación Campus de Vegazana, s/n. 24071 León, España mcrodl@unileon.es

\begin{abstract}
Resumen
Un código tipo surge de la potestad que tienen las instituciones para elaborar sus propias normas de régimen interno, de organización y funcionamiento, con el fin de llevar a cabo su misión y alcanzar los objetivos que tenga fijados. Como instrumento normativo al servicio de las entidades que lo elaboran, sirve para dotar de especiales cautelas el tratamiento de la protección de datos de carácter personal de los colectivos a los que sirve. El artículo se enfoca al sector privado.
\end{abstract}

\section{Palabras clave}

Código tipo, Protección de datos de carácter personal, Normativa sectorial, Empresa privada, Derecho a la información.

Title: Type codes: right to information and personal data protection

\begin{abstract}
Type codes arise from institutions' authority to develop their own internal rules of organization and operation, with the aim of adapting to the needs and peculiarities of their services. As a policy instrument, type codes provide with special precautions in handling the protection of personal data. This paper seeks to inform all professionals, whether working in the body producing the documentation or in the archival destination, of the value of the measures taken in this regard within the private sector.
\end{abstract}

\section{Keywords}

Type codes, Protection of personal data, Sectorial policies, Private enterprises, Right to information.

Rodríguez-López, María-del-Carmen. "Códigos tipo: derecho a la información y protección de datos personales". El profesional de la información, 2012, septiembre-octubre, v. 21, n. 5, pp. 509-513.

http://dx.doi.org/10.3145/epi.2012.sep.10

\section{Introducción}

El derecho aspira a solucionar los conflictos que tienen lugar en la sociedad. Las normas contienen juicios de valor importantes que conectan de forma inmediata con lo que entendemos por justo o erróneo. Los problemas éticos preceden al derecho, pero la norma comporta siempre una decisión. La Ley orgánica 15/1999 de protección de datos de carácter personal (LOPD), de 13 de dic., es la norma básica que regula este aspecto y deroga la anterior Ley orgánica 5/1992 de regulación del tratamiento automatizado de datos (Lortad), de 24 de sept., y traspone la Directiva comunitaria 95/46/ $C E$, mientras que su desarrollo reglamentario se hizo a través del Real decreto 1720/2007, de 21 de dic. Su finalidad es "garantizar y proteger, en lo que concierne al tratamiento de los datos personales, las libertades públicas y los derechos fundamentales de las personas físicas y especialmente el derecho de su honor e intimidad personal y familiar".

El pluralismo ético de las sociedades civiles democráticas 
hace que las leyes tengan en cuenta los criterios dominantes o mayoritarios y a la vez, siempre que el interés general no sucumba, respeten las convicciones de la minoría. Por su parte, la legislación sectorial, dentro de los marcos normativos contradictorios en los que se ve inmersa, encuentra una vía de salida en los códigos de conducta. Las instituciones tienen, entre sus capacidades de auto-organización, la potestad de aprobar sus propias normas de régimen interno, organización y funcionamiento, con el fin de adaptarse a las necesidades y singularidades de su misión.

Para Martínez-Bargueño (1986, p. 57), bajo el nombre común de derecho ciudadano a la información administrativa pueden agruparse distintos tipos de derechos, entre ellos el control de los ciudadanos sobre la información que posee la Administración sobre ellos.

El derecho de acceso a los datos tiene que ir acompañado "por un derecho a la confidencia que entraña una limitación de la circulación de informaciones que no pueden ser comunicadas, tanto interna como externamente a la Administración, sobre la base del consentimiento escrito de la persona afectada". Para la protección de los derechos individuales a la intimidad personal y familiar y para la regulación del uso de los bancos de datos que obran en poder de la Administración, las legislaciones europeas y de otros continentes han seguido los principios básicos contenidos en el Convenio para la protección de las personas con respecto al tratamiento automatizado de datos de carácter personal de 28/01/1981 (Convenio de Estrasburgo, BOE, 1985).

La sociedad informatizada, con sus aplicaciones y medios electrónicos y telemáticos, ha generado nuevos derechos fundamentales a partir de otros derechos constitucionales, como sucede en la jurisprudencia española en su interpretación del artículo 18.4 de nuestra constitución ${ }^{1}$. La LOPD pretende compaginar el ejercicio y la protección de estos derechos con el uso de nuevas tecnologías informáticas y de comunicación. Con la web 2.0 el ciudadano participa como proveedor de imágenes e informaciones de todo tipo y de su propia persona en blogs, redes sociales u otros medios. Como estas tecnologías permiten monitorizar las conductas, los ciudadanos se ven sometidos al riesgo de que se vulnere su intimidad o se vea en peligro la confidencialidad de sus acciones.

\section{Metodología}

Este trabajo analiza las medidas adoptadas en este sentido en el sector privado. Se han estudiado los códigos tipo aprobados hasta el momento por la Agencia Española de Protección de Datos (AEPD), además del análisis bibliográfico y de la legislación que afecta al tema. No son muchos los códigos tipo inscritos hasta este momento $2: 12$ códigos de empresas sujetas a derecho privado, más 1 para entidades locales de la Asociación de Municipios Vascos. En este artículo se analizan los de las 12 empresas:

- Tratamiento de datos de carácter personal para establecimientos sanitarios privados de la provincia de Sevilla (en adelante S-Sevilla).

- Fichero de automóviles de pérdida total, robo e incendios (en adelante Automóviles)
- Farmaindustria.

- Veraz-Persus.

- Sector de la Intermediación Inmobiliaria AEGI.

- Asociación Catalana de Recursos Asistenciales (ACRA).

- Universidad de Castilla-La Mancha (UCLM)

- Odontólogos y Estomatólogos de España (en adelante O-E)

- Confianza On-Line.

- Unió Catalana d'Hospitals (en adelante Cat-Hospitals)

- Agrupación Catalana de Establecimientos Sanitarios (en adelante Cat-Sanitarios)

- Fichero histórico de seguros del automóvil (Unespa).

En el apartado de bibliografía se incluyen las referencias de los textos y la legislación básica y sectorial citada. No aparece con exhaustividad toda la legislación sectorial que fundamenta el articulado de los códigos tipo, pues ya está reflejada en ellos.

\section{Qué es un código tipo}

Es el instrumento normativo al servicio de las entidades que lo elaboran, concebido para dotar de especiales cautelas el tratamiento de datos de carácter personal de los colectivos a los que sirven en el ejercicio de sus actividades profesionales, sin entrar en conflicto de competencias o de contenidos con el resto del ordenamiento legal, autonómico o estatal ${ }^{3}$. Su carácter de voluntariedad y de código deontológico o de buena práctica profesional no impide que sea vinculante para aquel que se adhiera al mismo y lo convierta en su código de conducta.

Los códigos tipo están concebidos para adecuar las disposiciones generales de la $L O P D$ y el Reglamento de desarrollo de la ley orgánica de protección de datos de carácter personal (en adelante Rlopd) a la casuística de los colectivos profesionales o empresas": "Mediante acuerdos sectoriales, convenios administrativos o decisiones de empresa, los responsables de tratamientos de titularidad pública y privada, así como las organizaciones en que se agrupen, podrán formular códigos tipo que establezcan las condiciones de organización, régimen de funcionamiento, procedimientos aplicables, normas de seguridad del entorno, programas o equipos, obligaciones de los implicados en el tratamiento y uso de la información personal, así como las garantías, en su ámbito, para el ejercicio de los derechos de las personas con pleno respeto a los principios y disposiciones de la presente Ley y sus normas de desarrollo. Podrán contener o no reglas operacionales detalladas de cada sistema particular y estándares técnicos de aplicación..." (LOPD, art. 32).

Por ley (Rlopd, art. 73, 74 y 75) deben contener unos aspectos mínimos, sin perjuicio de otros de carácter potestativo. Estos aspectos mínimos incluyen:

- delimitación de su ámbito de aplicación;

- actividades a que el código se refiere y los tratamientos sometidos al mismo;

- establecimiento de estándares homogéneos para el cumplimiento por los adheridos al código;

- establecimiento de procedimientos que faciliten el ejercicio por los afectados de sus derechos de acceso, rectificación, cancelación y oposición;

- determinación de las cesiones y transferencias internacio- 
nales de datos que en su caso se prevean, con indicación de las garantías que deban adoptarse;

- previsiones específicas para la aplicación de los principios de protección de datos;

En el código se refuerza la protección de los derechos ARCO (acceso, rectificación, cancelación y oposición)

- acciones formativas dirigidas a quienes los traten;

- mecanismos de supervisión que garanticen el cumplimiento por los adheridos de lo establecido en el código tipo.

En particular deberán contenerse en el código aquellos mecanismos que refuercen la protección de los derechos ARCO (derechos de acceso, rectificación, cancelación y oposición):

- Cláusulas tipo para la obtención del consentimiento de los afectados al tratamiento o cesión de sus datos.

- Cláusulas tipo para informar a los afectados del tratamiento, cuando los datos no sean obtenidos de los mismos.

- Modelos para el ejercicio por los afectados de sus derechos de acceso, rectificación, cancelación y oposición.

- Modelos de cláusulas para el cumplimiento de los requisitos formales exigibles para la contratación de un encargado del tratamiento, en su caso.

- Procedimientos de supervisión independientes para garantizar el cumplimiento de las obligaciones asumidas por los adheridos, y establecer un régimen sancionador adecuado, eficaz y disuasorio.

- Podrán incluir otro contenido potestativo ${ }^{5}$.

Los códigos tipo registrados en España constan generalmente de dos partes. La primera desgrana las normas o el código de conducta en sí, partiendo de una justificación de los motivos que llevan a adoptarlos. La segunda parte incluye los mecanismos de control y seguimiento del proceso. En buen número de ellos se incluye como tercera parte el sello destinado a acreditar la adhesión al sistema de autorregulación de forma visual (ACES, AEGI, Confianza On-Line, O-E, Cat-Hospitals, Veraz-Persus).

\section{Objetivos de un código tipo}

El redactado de normas, pautas o códigos de conducta trata de dar respuesta al requerimiento legal. Que un colectivo se movilice al respecto y otro no, responde a motivaciones diferentes.

Los objetivos de un código tipo son:

- Concienciar a los centros adheridos sobre la importancia y la obligatoriedad de esta materia, y sensibilizarlos en la guarda de la máxima confidencialidad en todo lo referente a la intimidad de los clientes (ACRA, S-Sevilla).

- Educar al consumidor sobre sus derechos y la manera de protegerlos, facilitándole el ejercicio de sus derechos y la defensa de sus intereses (AEGI, Farmaindustria, Cat-Hospitals).

- Uniformizar las prácticas de todos los centros asociados: los centros y establecimientos fueron adaptando sus sis- temas informáticos y bases de datos a las prescripciones de la Lortad y de su Reglamento. El final del proceso lleva a un sistema de autorregulación integral (ACES, Confianza On-Line, Farmaindustria, S-Sevilla, Veraz-Persus).

- Proporcionar seguridad jurídica a los clientes: la uniformidad del régimen de protección de datos de carácter personal en el seno de cualquier agrupación evita la proliferación de diversos procedimientos o regímenes de protección y ejercicio de sus derechos. Este objetivo otorga valor añadido tanto a los consumidores como a la industria (ACRA, AEGI, Confianza On-Line, O-E, Unespa, Cat-Hospitals, Veraz-Persus).

- Mejorar la imagen de marca, incluyendo como característica de calidad homogénea del colectivo el esfuerzo corporativo y la sensibilización del grupo o sector para garantizar el respeto a los derechos en el tratamiento de los datos personales de sus usuarios o clientes, ya sean pacientes, residentes, consumidores, etc. Como tal habrá un símbolo, marca o logotipo que exprese la existencia del código tipo y su aplicación y vigencia en los centros asociados; un sello de confianza (ACES, ACRA, y AEGI).

\section{Consentimiento del cliente para el tratamiento de sus datos personales}

En función del espectro profesional al que pertenecen los códigos tipo estudiados resulta aconsejable citar qué aspectos constituyen el ámbito de aplicación de protección de datos. La $\angle O P D$, asistida por diferentes sentencias del Tribunal Supremo o por la propia $A E P D$, permite entender que no se aplicará a las personas jurídicas. En el caso que nos ocupa: una empresa o persona jurídica, o autónomo o personal de contacto de la misma (entendiendo por tales las personas que ocupan un cargo directivo). Tampoco se aplicará a los datos de fallecidos, a los procedimientos de disociación, libros de bautismos y ficheros de juzgados o tribunales. Sí se aplicará a datos de menores de edad, la imagen, videovigilancia, dirección IP, dirección e-mail y los datos biométricos (Garrido-Falla, 2012, p. 2829).

La $L O P D$ amplió el ámbito de protección de los datos de carácter personal a todo tipo de soporte. En su disposición adicional primera establece plazos distintos para la adecuación de los ficheros preexistentes a sus prescripciones, y para el cumplimiento de la obligación de comunicar su existencia a la $A E P D$, según sean automatizados o no ${ }^{6}$. Los ficheros automatizados inscritos o no en el Registro General de Protección de Datos debieron adecuarse a la Ley antes del 14 de enero de 2003. Los ficheros no automatizados tuvieron como fecha límite el 24 de octubre de 2007. Adaptarse a la normativa vigente actualizó las condiciones de organización, régimen de funcionamiento, procedimientos aplicables, normas de seguridad del entorno, programas o equipos, obligaciones de los implicados en el tratamiento y uso de la información personal, así como el régimen de garantías. Todo ello sin perjuicio de los derechos ARCO por parte de los afectados, que podrán ejercerlos sin esperar ningún plazo, pues son los que la legislación general protege y aparecen unívocamente vinculados en los códigos tipo al "cliente", "usuario", "paciente", "asegurado", "estudiante"... Ese es el motivo de que en este 
trabajo no se traten los derechos ARCO, y sí el punto que obligó a la reelaboración de todos los códigos ya registrados antes de 2007: la recogida leal y lícita de los datos con el consentimiento del cliente.

Las cláusulas tipo se establecen para la obtención del consentimiento de los afectados al tratamiento o cesión de sus datos y para informarles cuando los datos se han obtenido por otro medio. En caso de que los datos de carácter personal no hayan sido recabados del titular, éste deberá ser informado de forma expresa, precisa e inequívoca de la procedencia de los mismos. Además se recabará su consentimiento para el tratamiento de los datos personales que le conciernen y se le facilitarán los medios para revocar su consentimiento si hay causa justificada. Todos los códigos tipo prohíben la recogida de datos personales por medios fraudulentos, desleales o ilícitos. Además, establecen que aquellas entidades adheridas al código que recaben datos personales por cualquier método, incluidos los medios electrónicos de comunicación a distancia, deberán informar previamente a los titulares, de forma inequívoca y claramente perceptible:

- de la existencia de un fichero o tratamiento de datos de carácter personal y de la finalidad de su recogida, así como de los destinatarios de la información;

- de la identificación del responsable del fichero inscrito en el Registro de la AEPD con su dirección postal y de correo electrónico, que facilite la comunicación para poder ejercer los derechos ARCO;

- del carácter obligatorio o facultativo de la respuesta a las preguntas que en su caso les sean planteadas, así como de las consecuencias de la obtención de los datos o la negativa a suministrarlos.

En los casos que así se requiera -por ej. en centros ocupados en la salud-se explicita a clientes o usuarios que tienen el deber de facilitar los datos sobre su estado físico o sobre su salud de manera leal y verdadera, así como colaborar en su obtención, especialmente cuando sean necesarios por razones de interés público o con motivo de la prestación de determinados servicios.

Hay que distinguir los supuestos en que el tratamiento tiene por objeto meros datos personales, de cuando lo constituyen los relativos a la salud, pues estos últimos están sujetos a un régimen especial?:

- por una parte los datos recabados en el establecimiento de la relación jurídico-contractual existente con el usuario;

- por otra los necesarios para la prestación de los servicios asistenciales y que incluye la información relativa a la salud del residente o usuario.

Este tipo de información puede darse también en instituciones no sanitarias. Por ejemplo en la gestión inmobiliaria un cliente puede haber proporcionado datos relativos a su salud para indicar aquellas características que habrá de cumplir el inmueble. En este caso sólo se harán constar estas últimas, pero no el dato de la salud del cliente.

La información sobre las medidas adoptadas por los centros adheridos deberá facilitarse de forma comprensible y adecuada al cliente o usuario de los servicios, recomendándose su inclusión de forma clara y legible en los cuestionarios, impresos o documentos que se utilicen para recoger datos. Los códigos al cuidado de la salud buscan un procedimiento de mayor alcance, acudiendo a la señalética con carteles ubicados al alcance del usuario.

\section{Conclusiones}

Cualquier profesional de la información, y especialmente el encargado del tratamiento de la documentación de un archivo, debe conocer la existencia de los códigos tipo y de sus pautas para la gestión del acceso a la información y al documento de archivo. La profesión de archivero se ve afectada cada vez con mayor frecuencia por la colisión entre los derechos de acceso y los derechos de protección de la intimidad de las personas. Tanto la legislación como la normativa sectorial han inclinado la balanza hacia la restricción al acceso frente a la difusión, lo cual ha afectado gravemente a los usuarios que necesitan consultar los archivos.

El punto en que se concentra una buena parte de la inquietud de cualquier institución privada es el consentimiento de los afectados al tratamiento o cesión de sus datos y la elaboración del mecanismo ideal para informarlos del tratamien-

Cualquier profesional de la información, y especialmente el archivero, debe conocer la existencia de los códigos tipo

to, cuando los datos no sean obtenidos de los mismos. Si han sido proporcionados por terceros el interesado deberá ser informado de forma expresa, precisa e inequívoca de su procedencia. Los códigos tipo incluyen en el texto el formulado de estas cláusulas, y en sus anexos el modelo de documento de consentimiento que ha de firmar el interesado.

Internet y sus posibilidades tecnológicas han producido una inmersión del usuario en el mundo virtual. El atractivo de la interactuación usuario-usuario y la posibilidad de ofrecer opiniones e informaciones en el momento, ha supuesto un cambio tan drástico que se podría hablar de cambio de naturaleza del proveedor de información y de datos de carácter personal. El propio dueño del dato sirve de proveedor. Si bien la $A E P D$ recoge en su articulado y en sucesivos informes las pautas al respecto, este cambio de naturaleza hace necesaria una mayor adecuación en el sector. Es especialmente necesaria la adopción de medidas destinadas a facilitar al usuario el ejercicio de sus derechos reconocidos por la normativa de protección de datos.

\section{Notas}

1. “La ley limitará el uso de la informática para garantizar el honor y la intimidad personal y familiar de los ciudadanos y el pleno ejercicio de sus derechos".

\section{Agencia Española de Protección de Datos (AEPD). http://www.agpd.es}

3. El Convenio, en su art. 11 permite establecer una protección más amplia: "Ninguna de las disposiciones del presente capítulo se interpretará en el sentido de que limite la facul- 
tad, o afecte de alguna otra forma a la facultad de cada parte, de conceder a las personas concernidas una protección más amplia que la prevista en el presente Convenio".

4. La elaboración de los códigos tipo aparece en el Rlopd, art. 71, 72 iniciativa y ámbito de aplicación, 73 y 75.1 y 2 contenido preceptivo y en la $\angle O P D$, art. 32.

5. Agencia Española de Protección de Datos (AEPD). Elaboración de códigos tipo.

https://www.agpd.es/portalwebAGPD/canalresponsable/ elaboracion_codigos_tipo/index-ides-idphp.php

6. El Reglamento de medidas de seguridad de los ficheros automatizados que contengan datos de carácter personal, Real decreto 994/1999, en su disposición transitoria única, modificada por el Real decreto 195/2000, había establecido los siguientes plazos para la implantación de las medidas de seguridad en los sistemas de información automatizados que se encontraban en funcionamiento a su entrada en vigor: medidas de seguridad de nivel básico: 26 de marzo de 2000; medidas de seguridad de nivel medio: 26 de junio de 2000; medidas de seguridad de nivel alto: 26 de junio de 2002.

7. Entre la variada casuística revisada destacan algunas de las cautelas especiales, entre ellas la establecida por Amcra (código tipo, p. 5): "Nuestros asociados disponen de información personal relativa a la salud de sus residentes o usuarios. Esta información merece la máxima confidencialidad y protección, no sólo por referirse a una de las esferas más íntimas de la persona física, como es su salud, sino también por el hecho de pertenecer a un sector de la población, el de las personas en situación de dependencia, que precisa de una especial protección, ya que en muchas ocasiones se encuentran en una situación que les impide conocer y ejercitar sus derechos en igualdad de condiciones. En el tratamiento de los datos de carácter personal de los residentes o usuarios tiene especial importancia la cuestión de la incapacidad declarada o no por sentencia judicial de éstos".

\section{Bibliografía}

Convenio para la protección de las personas con respecto al tratamiento automatizado de datos de carácter personal, hecho en Estrasburgo el 28 de enero de 1981. BOE n. 274, de 15 de nov. de 1985, pp. 36000-36004.

http://www.boe.es/buscar/doc.php?id=BOE-A-1985-23447

Ley 16/1985, de 25 de junio, del Patrimonio histórico español. BOE n. 155, de 29 de junio de 1985.

http://www.mcu.es/patrimonio/docs/ley16-1985.pdf

Ley 14/1986, de 25 de abril, General de sanidad. BOE n. 101, de 29 de abril de 1986.

http://www.boe.es/buscar/act.php?id=BOE-A-1986-10499

Ley 34/2002, de 11 de julio, de Servicios de la Sociedad de la información y de comercio electrónico. BOE n. 166, de 12 de julio de 2002.

http://www.boe.es/boe/dias/2002/07/12/pdfs/A2538825403.pdf

Ley 41/2002, de 14 de noviembre, básica reguladora de la autonomía del paciente y de derechos y obligaciones en materia de información y documentación clínica. BOE n. 274, de 15 de noviembre de 2002.

http://www.boe.es/boe/dias/2002/11/15/pdfs/A4012640132.pdf

Real Decreto 1720/2007, de 21 de diciembre, por el que se aprueba el Reglamento de Desarrollo de la Ley Orgánica 15/1999, de 13 de diciembre, de Protección de datos de carácter personal. BOE n. 17, de 19 de enero de 2008, pp. 4103-4136.

http://www.boe.es/boe/dias/2008/01/19/pdfs/A0410304136.pdf

Garrido-Falla, Carlos. "Protección de datos y acceso a ficheros públicos". Tábula, 2012, n. 15, pp. 23-42.

Martínez-Bargueño, Manuel. Teoría y práctica de la información administrativa al ciudadano. Madrid: Instituto Nacional de Administración Pública, 1986.

\section{EPI $n^{\circ} 1$ en el ranking In-Recs Anuario ThinkEPI en $8^{\text {a }}$ posición}

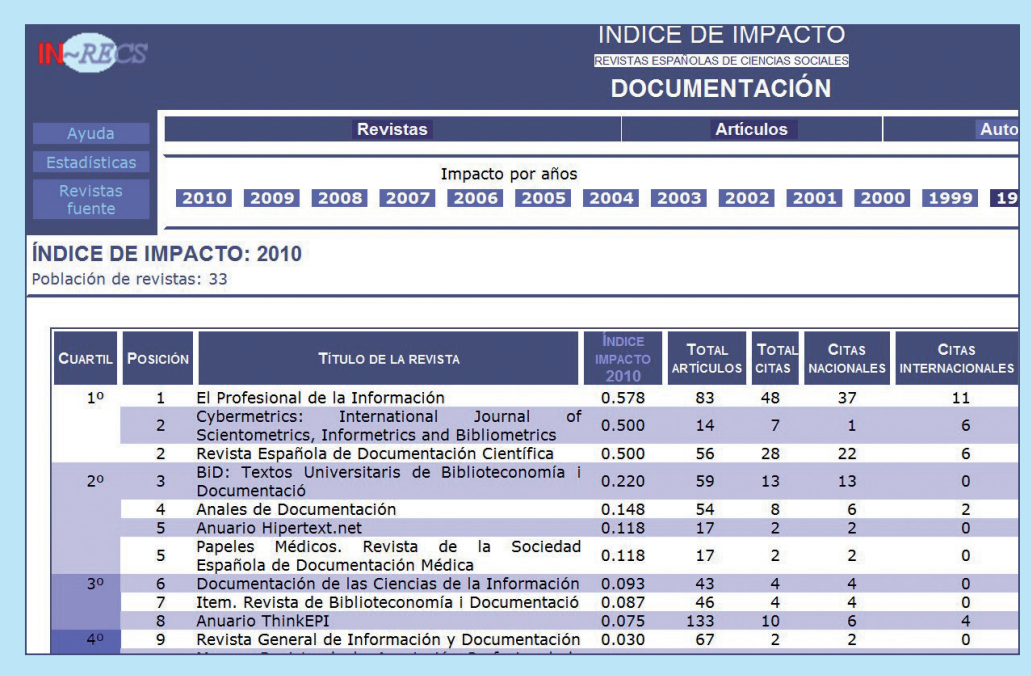

Según el Índice de Revistas Españolas de Ciencias Sociales (In-Recs), elaborado por el Grupo EC3 (Evaluación de la Ciencia y de la Comunicación Científica) de la Universidad de Granada:

http://ec3.ugr.es/in-recs/ii/ Documentacion-2010.htm la revista El profesional de la información ha conseguido situarse en primer lugar, de entre 33 revistas españolas de biblioteconomía y documentación. 